\title{
Band alignment at GaN/single-layer WSe2 interface
}

\author{
Malleswararao Tangi ${ }^{1}$, Pawan Mishra ${ }^{1}$, Chien-Chih Tseng ${ }^{2}$, Tien Khee $\mathrm{Ng}^{1}$, Mohamed Nejib \\ Hedhili ${ }^{3}$, Dalaver H. Anjum ${ }^{3}$, Mohd Sharizal Alias ${ }^{1}$, Nini $\mathrm{Wei}^{3}$, Lain-Jong $\mathrm{Li}^{2}$, and Boon S. \\ $\mathrm{Ooi}^{1 *}$ \\ ${ }^{1}$ Photonics Laboratory, Computer, Electrical, and Mathematical Sciences and Engineering \\ (CEMSE) Division, King Abdullah University of Science and Technology (KAUST), Thuwal \\ 23955-6900, Saudi Arabia. \\ ${ }^{2}$ Physical Science and Engineering (PSE) Division, King Abdullah University of Science and \\ Technology (KAUST), Thuwal 23955-6900, Saudi Arabia. \\ ${ }^{3}$ Imaging and Characterization Laboratory, King Abdullah University of Science and \\ Technology (KAUST), Thuwal 23955-6900, Saudi Arabia.
}

\begin{abstract}
We study the band discontinuity at the GaN/single-layer (SL) WSe 2 heterointerface. The GaN thin layer is epitaxially grown by molecular beam epitaxy on chemically vapor deposited $\mathrm{SL}-\mathrm{WSe} / \mathrm{c}$-sapphire. We confirm that the $\mathrm{WSe}_{2}$ was formed as an SL from structural and optical analyses using atomic force microscopy, scanning transmission electron microscopy, microRaman, absorbance, and micro-photoluminescence spectra. The determination of band offset parameters at the GaN/SL-WSe 2 heterojunction is obtained by high-resolution $\mathrm{x}$-ray photoelectron spectroscopy, electron affinities and the electronic bandgap values of SL-WSe 2 and GaN. The valence band and conduction band offset values are determined to be $2.25 \pm 0.15$ and $0.80 \pm 0.15 \mathrm{eV}$ respectively, with type II band alignment. The band alignment parameters determined here provide a route towards the integration of group III nitride semiconducting materials with transition metal dichalcogenides (TMDs) for designing and modeling of their heterojunction based electronic and optoelectronic devices.
\end{abstract}

KEYWORDS: GaN, single layer $\mathrm{WSe}_{2}, 3 \mathrm{D} / 2 \mathrm{D}$ heterojunction, HRXPS, band alignment, molecular beam epitaxy

*Corresponding author electronic mail: boon.ooi@kaust.edu.sa 


\section{INTRODUCTION}

Group-III nitrides, GaN as a primary material, are well demonstrated with enormous applications in high efficiency electronic and optoelectronics devices such as high electron mobility transistors, light emitting diodes, and laser diodes. ${ }^{1-4}$ Recently, group VI transition metal dichalcogenides (TMDs) in the form of $\mathrm{MX}_{2}$ has emerged as a novel atomic layered material system with challenging thermoelectric, electronic and optoelectronic properties. ${ }^{5-8}$ Among the TMDs, tungsten diselenide ( $\mathrm{WSe}_{2}$ ) in a single layer form is of potential interest for such devices owing to its direct bandgap of $\approx 1.65 \mathrm{eV}$ and prominent transport properties. ${ }^{9,10} \mathrm{GaN}$ has small lattice mismatch of $\approx 3.2 \%, \approx 1.0 \%$ and $\approx 0.8 \%$ with majorly studied TMDs such as $\mathrm{WSe}_{2}$, tungsten disulfide $\left(\mathrm{WS}_{2}\right)$ and molybdenum disulfide $\left(\mathrm{MoS}_{2}\right)^{11}$, respectively, in comparison to commonly used substrates such as sapphire, $\mathrm{SiC}$ and $\mathrm{Si}^{12,13}$ Moreover, recent advances in the integration of 2D-layered materials with wide band gap group III nitride semiconductors is exciting due to their variety of applications in high current tunnel diodes. ${ }^{14,15}$ Several efforts were made to grow $\mathrm{GaN}$ on closely lattice matched TMDs. Yamada et al. presented the growth of $\mathrm{GaN}$ on bulk $\mathrm{MoS}_{2}$ by plasma-enhanced molecular beam epitaxy ${ }^{16}$. There were recent attempts to grow $\mathrm{GaN}$ on large area $\mathrm{MoS}_{2}$ and $\mathrm{WS}_{2}$ layers, ${ }^{11,17}$ layered $\mathrm{MoS}_{2}$ on $\mathrm{GaN}$ epilayers ${ }^{18}$ by chemical vapor deposition (CVD) growth techniques and layer transferred $\mathrm{p}-\mathrm{MoS}_{2}$ on $\mathrm{GaN}^{14}$. Though the deposition of large area single-layer $\mathrm{WSe}_{2}$ on sapphire exhibiting direct bandgap has been demonstrated, ${ }^{9,19}$ the growth of $\mathrm{GaN}$ on such large area monolayered $\mathrm{WSe}_{2}$ has not yet been explored.

So far, the band offset parameters were determined for either group III nitrides on various other semiconducting materials or solely TMDs based dissimilar heterostructures using X-ray photoemission spectroscopic core-levels evaluated with respect to the valence band maximum and bandgap studies. Though, the band offset parameters (junction type: valence band offset- $\Delta \mathrm{E}_{\mathrm{v}}$ \& conduction band offset- $\Delta \mathrm{E}_{\mathrm{c}}$ ) are measured for various heterojunctions in the literature. ${ }^{20-25}$ Recently we have reported the growth of GaN on SL-MoS 2 and the band alignment parameters (Type-II: $1.86 \pm 0.08 \& 0.56 \pm 0.10 \mathrm{eV}$ ) for GaN/SL-MoS 2 hetero-interface. ${ }^{26}$ The band offset parameters (valence band offset $(\mathrm{VBO})-\Delta \mathrm{E}_{\mathrm{v}}$ and conduction band offset $(\mathrm{CBO})-\Delta \mathrm{E}_{\mathrm{c}}$ ) and type of junction by HRXPS for epitaxially formed GaN/SL-WSe ${ }_{2}$ hetero-interface is yet to be experimentally investigated. The determination of band offset parameters is required to study the group III nitride/TMDs heterojunction based devices. These hybrid heterojunctions, exhibiting type I and II band alignments, can be utilized as TMD quantum well based light emitting devices 
and the tunnel diodes, respectively. ${ }^{6,15}$ Thus, the growth of GaN/SL-WSe 2 and the determination of band offsets are necessary to provide a route towards the integration of group III nitrides with TMDs for designing the electronic and optoelectronic devices.

In this report, in order to study the band discontinuity at the GaN/SL-WSe 2 heterointerface, thin GaN layers were epitaxially grown on a CVD prepared SL-WSe2. The sustainability of SL$\mathrm{WSe}_{2}$ during GaN growth is confirmed by the atomic force microscopy (AFM), microphotoluminescence $(\mu \mathrm{PL})$ and Raman spectroscopies. In addition, the band offset parameters for $\mathrm{GaN}_{\text {SL-WSe}}$ heterojunction were determined using high-resolution $\mathrm{x}$-ray photoelectron spectroscopy (HRXPS) and electronic band gap values of respective constituent layers in the heterojunction.

\section{EXPERIMENTAL SECTION}

The growth experiments of $\mathrm{GaN}$ on $\mathrm{WSe}_{2} / \mathrm{c}$-sapphire substrates were carried out by Veeco 930 Gen plasma assisted molecular beam epitaxy (PAMBE) system at substrate temperature of $500{ }^{\circ} \mathrm{C}$. The large area $\mathrm{WSe}_{2}$ SLs were prepared on c-sapphire substrates using CVD and the details can be found elsewhere ${ }^{19}$. The ion and a cryo pumps were employed to attain a base pressure of $3 \times 10^{-11}$ Torr and oxygen partial pressure $<10^{-11}$ Torr, as obtained by a residual gas analyzer (RGA). The substrates were thermally outgassed in introduction chamber at $200{ }^{\circ} \mathrm{C}$ for 30 mins, further cleaning was carried out in preparation chamber at $300{ }^{\circ} \mathrm{C}$ for $60 \mathrm{mins}$ and in the growth chamber at $400{ }^{\circ} \mathrm{C}$ for 30 mins. For GaN growth, the nitrogen plasma source was operated with the flow rate of 1 standard cubic centimeter per minute $(\mathrm{sccm})$ and RF power of $300 \mathrm{~W}$ and Ga metal was evaporated by standard Knudsen cell with beam equivalent pressure (BEP) value of $2.10 \times 10^{-8}$ Torr. Prior to this, thin AlN buffer layer was grown. The corresponding chamber pressure was $\approx 2.5 \times 10^{-5}$ Torr. The thickness of the GaN epilayer (sample C) is measured to be $\approx 500 \mathrm{~nm}$. The thickness of $\mathrm{GaN}$ layer in sample B was estimated to be $6 \pm 1 \mathrm{~nm}$ from growth rate calibrations. The Agilent technologies 5400 atomic force microscopy was used in tapping mode to acquire the surface morphology of the samples. Structural quality of $\mathrm{GaN}$ epilayer was investigated by $\mathrm{CuK}_{\alpha}$ High Resolution X-ray Diffraction (HRXRD) having four-bounce Ge(022) monochromator. Highangle annular dark field scanning transmission electron microscopy (HAADF-STEM) was utilized by operating a probe-corrected FEI Titan at an acceleration voltage of $300 \mathrm{kV}$. A cross- sectional TEM specimen of sample B was prepared by using FEI's Helios Dual Beam focused ion beam 
(FIB)/SEM equipped with an Omniprobe. Electron energy loss spectroscopy (EELS) acquisition was performed by using Gatan's GIF Quantum of Model 966. Using Horiba Aramis room temperature (RT) $\mu \mathrm{PL}$, the emission of $\mathrm{GaN}$ and $\mathrm{WSe}_{2}$ layers was deduced with excitation lines of He-Cd laser of $325 \mathrm{~nm}$ and He-Ne laser of $633 \mathrm{~nm}$, respectively. Absorbance spectra were acquired with a Shimadzu UV3600 spectrophotometer equipped with integrating sphere. The highresolution XPS measurements were carried out using a Kratos Axis Ultra DLD spectrometer equipped with a monochromatic $\mathrm{Al} \mathrm{K} \alpha \mathrm{X}$-ray source $(\mathrm{h} v=1486.6 \mathrm{eV})$ operating at $150 \mathrm{~W}$, a multichannel plate and delay line detector under a vacuum of $\sim 10^{-9}$ mbar. The high-resolution spectra were collected within the limits of spatial resolution at a fixed analyzer pass energy of $20 \mathrm{eV}$. In order to eliminate the shifts in the HRXPS spectra associated with the surface charging effect, the measurements were acquired both with and without electron beam charge compensation. For both cases, no changes were observed in the determined band alignment. In absence of electron beam charge, the surface of samples was electrically connected with a clean copper $(\mathrm{Cu})$ foil. The remnant binding energy shifts were referenced to the adventitious carbon $(\mathrm{C} 1 \mathrm{~s})$ signal. ${ }^{27-29}$ In this study, CVD grown SL-WSe 2 (sample A), MBE grown GaN on SL-WSe 2 (sample B) and GaN epilayer (sample C) were used to determine the band offsets at GaN/SL-WSe 2 hetero-interface.

\section{RESULTS AND DISCUSSION}

To investigate the surface morphology, root mean square (rms) roughness of GaN/WSe2 (B) and $\mathrm{GaN}$ epilayer (C), and thickness of $\mathrm{WSe}_{2}$ layer (A), AFM scans were carried out in tapping mode. Figures 1(a-c) show the AFM surface topography scans collected on samples C, A and B, respectively. The AFM images were obtained at different scales to view the fine features according to surface smoothness of the formed films. Color bars on top of the respective images in Figure 1 show height contrast of the features. From Figure 1(a), the root mean square (rms) surface roughness for GaN film is $\approx 2 \mathrm{~nm}$. Moreover, AFM is one of the most commonly used technique to determine the number of monolayers of TMDs samples. The thickness of the large area $\mathrm{WSe}_{2}$ layer is found to be $\approx 0.76 \mathrm{~nm}$ from the line profile as shown in Figure 1(b) for sample A, which is in agreement with the thickness of Se-W-Se single-layer. ${ }^{19}$ The inset to Figure 1(b) displays the optical microscopy image of large area WSe 2 layer. Figure 1(c) shows the AFM surface morphology for sample B exhibiting surface rms roughness of $\approx 4 \mathrm{~nm}$ which is higher than that of relaxed $\mathrm{GaN}$ epilayer (sample $\mathrm{C}$ ) that results from the lattice mismatch between thin $\mathrm{GaN}$ and 
$\mathrm{WSe}_{2}$. Thus, AFM study confirms that the CVD deposited large area $\mathrm{WSe}_{2}$ is in the form of a single layer. Further, HRXRD measurements were performed to investigate the crystalline structure and quality of the GaN epilayer (sample C). Figure 1(d) presents the $2 \theta-\omega$ scans, which were obtained on the symmetric (on-axis) and asymmetric (off-axis) planes of sample C. Asymmetric planes were studied in skew symmetric geometry. ${ }^{4}$ Figure $1(\mathrm{~d})$ displays c-oriented peaks: at $\mathrm{GaN}(0002)$ at $34.52^{\circ}, \mathrm{GaN}(0004)$ at $73.20^{\circ}$ along with the buffer layer $\mathrm{AlN}(0002)$ at $35.89^{\circ}$ and $\mathrm{Al}_{2} \mathrm{O}_{3}$ substrate (0006) at $41.45^{\circ}$, respectively. The asymmetric reflection shows $\mathrm{GaN}($ $10 \overline{1} 1)$ peak at $36.81^{\circ} \operatorname{GaN}(20 \overline{2} 2)$ at $78.77^{\circ}$ along with the buffer layer peak $\mathrm{AlN}(10 \overline{1} 1)$ at $38.50^{\circ}$ and substrate peak $\mathrm{Al}_{2} \mathrm{O}_{3}(11 \overline{2} 3)$ at $43.08^{\circ}$, respectively. The respective inset shows the $\omega$ scans for on-axis $\mathrm{GaN}(0002)$ and off-axis $\mathrm{GaN}(10 \overline{1} 1)$ reflections, with broadening values of $\approx 350$ and $\approx 980$ arc-sec, respectively. Thus, from HRXRD analysis, sample $\mathrm{C}$ possess single crystalline wurtzite structure grown along c-axis exhibiting moderate crystal quality.

HAADF-STEM studies were performed on sample B (GaN/WSe $2 / \mathrm{c}$-sapphire) to confirm the existence and the thickness of $\mathrm{GaN}$ and $\mathrm{WSe}_{2}$ layers. Figure 2(a) shows the cross sectional HAADF-STEM image recorded on sample B which is prepared by FIB. Prior to this, the Ti metal layer having thickness of $\approx 500 \mathrm{~nm}$ was deposited on sample B by e-beam evaporation method to avoid any physical damage of thin $\mathrm{GaN}$ and $\mathrm{WSe}_{2}$ layers at the heterojunction caused by focused ion beams while the sample preparation was done for STEM studies. This reveals that the thickness of $\mathrm{GaN}_{\mathrm{WSe}}$ heterojunction is $\approx 7 \mathrm{~nm}$. Figure 2(b) shows the magnified STEM image of Figure 2(a) obtained across the GaN/WSe 2 /c-sapphire heterojunction (sample B). STEM-EELS imaging data sets were acquired to generate the elemental maps of $\mathrm{W}, \mathrm{Se}, \mathrm{Ga}$ and $\mathrm{Al}$ elements by employing W-M (1809 eV), Se-L (1436 eV), Ga-L (1117 eV), and Al-K (1560 eV) EELS edges, respectively. The elements $\mathrm{W}, \mathrm{Se}, \mathrm{Ga}$ and $\mathrm{Al}$ across the junction are, respectively, represented by Blue, Green, Red, and Yellow colored pixels. Figure 2(c) depicts the elemental mapping of heterojunction showing the existence of both $\mathrm{W}$ and Se atoms at the interface. A STEM-EDS line profile was generated to re-confirm the presence of SL-WSe2 that is sandwiched between sapphire substrate and GaN thin film. Figure 2(d) represents the EDS line profiles obtained for elements Al, Ga, W and Se across the junction. The intensity of line profiles for $\mathrm{W}$ and Se atoms increases in the region of SL-WSe 2 whereas the line profiles for $\mathrm{Ga}$ and $\mathrm{Al}$ elements exhibit high intensity in the regions of overgrown GaN and underlying sapphire substrates, respectively, as shown in Figure 2(d). Therefore, it is 
noted that the line profiles in Figure 2(d) were in accordance with the respective elemental color mapping as depicted in Figure 2(c). Thus, Figures 2 (b-d) depict the existence of $\mathrm{WSe}_{2}$ in the form of single layer having thickness of $\approx 0.78 \mathrm{~nm}$ which is in corroboration with AFM analysis. Overall, the HAADF-STEM analysis is a clear evidence of the formation of GaN/SL-WSe 2 heterojunction.

Micro-Raman spectroscopy was used to investigate the optical quality as well as to ensure that the CVD grown $\mathrm{WSe}_{2}$ is an SL. Figure 3(a-d) shows the Raman spectra for $\mathrm{WSe}_{2} / \mathrm{c}$-sapphire, GaN/WSe $2 /$-sapphire and GaN/c-sapphire. In order to obtain the enhanced Raman signal from $\mathrm{GaN}$ and $\mathrm{WSe}_{2}$ layers, the measurements were carried out with excitation lines of 325 and $633 \mathrm{~nm}$ by which signal can overcome the reduced resonant excitation effect. ${ }^{26,30}$ Thus, both the excitation sources are required to collect the high intensity phonon modes from the individual layer in GaN/SL-WSe 2 heterojunction as described in Figure 3(b and c). The two characteristic peaks in Figure 3(a and b) observed at $248 \mathrm{~cm}^{-1}$ and $259 \mathrm{~cm}^{-1}$ are attributed to $E_{2 g}^{1}$ and $\mathrm{A}_{1 \mathrm{~g}}$ phonon modes. ${ }^{10,19}$.These modes correspond to the in plane vibration of $\mathrm{W}$ and Se atoms $\left(E_{2 g}^{1}\right)$ and out of plane vibration of Se atoms $\left(A_{1 g}\right)$ in $\mathrm{WSe}_{2}$. The intensity ratio $\left(\mathrm{I}_{\mathrm{A}} / \mathrm{I}_{\mathrm{E}}\right)$ of these modes is higher than the reported values which can be attributed to the variation in doping levels. ${ }^{31}$ The observed peaks at 222, 241, 377 and $398 \mathrm{~cm}^{-1}$ stem from the WSe 2 layer which are consistent with the previous studies. ${ }^{10,32}$ The peaks represented by asterisks are from the sapphire substrate. The absence of a peak at $\approx 307 \mathrm{~cm}^{-1}$ confirms that the formed $\mathrm{WSe}_{2}$ is a single layer which is corroborated by AFM studies. These results also show the sustainability of $\mathrm{WSe}_{2}$ layer during the growth of $\mathrm{GaN}$ in PAMBE. In consequence of the reduced resonant excitation effect ${ }^{26}$, in Figure 3(b), $E_{2}^{H}$ mode of GaN exhibits lower intensity than the $E_{2 g}^{1}$ and $\mathrm{A}_{1 \mathrm{~g}}$ phonon modes of $\mathrm{WSe}_{2}$ while the sample $\mathrm{B}$ is excited with $633 \mathrm{~nm}$ line. In contrast, in Figure 3(c), the intensity of $E_{2}^{H}$ mode for GaN is higher than that of the phonon modes $\left(E_{2 g}^{1}\right.$ and $\mathrm{A}_{1 \mathrm{~g}}$ ) of $\mathrm{WSe}_{2}$ with $325 \mathrm{~nm}$ excitation. Further, Figure 3(c) shows a low intensity single peak for both $E_{2 g}^{1}$ and $\mathrm{A}_{1 \mathrm{~g}}$ phonon modes which is in good agreement with the literature. ${ }^{33-35}$ In Figure 3(b and c), the $E_{2}^{H}$ peak of thin GaN grown on WSe 2 is significantly blue shifted from that of the bulk GaN (sample C) having the peak at $568.5 \pm 0.5$ $\mathrm{cm}^{-1}$ which results from the strain accompanied by the pseudomorphic growth of GaN on SL$\mathrm{WSe}_{2} / \mathrm{c}$-sapphire. Thus, AFM, STEM and micro-Raman measurements confirm that the existence of SL-WSe 2 during the growth of GaN. 
In order to investigate the optical quality of both $\mathrm{WSe}_{2}$, bulk like $\mathrm{GaN}$ epilayer and GaN/WSe 2 (samples A-C) room temperature $\mu \mathrm{PL}$ and absorbance measurements were performed. Figure 4 ( $\mathrm{a}$ and $\mathrm{b}$ ) show the RT $\mu \mathrm{PL}$ and optical absorbance spectra acquired on GaN epilayer and $\mathrm{WSe}_{2}$. The optical band edge emissions ( $E_{\text {opt }}^{G a N}$ and $E_{\text {opt }}^{S L-W e_{2}}$ ) for $\mathrm{GaN}$ and $\mathrm{WSe}_{2}$ are observed at $\approx 3.43$ and $\approx 1.63 \mathrm{eV}$, respectively. The low intensity PL for sample B could be due to the presence of octahedral phase of $\mathrm{WSe}_{2}$, which will be discussed later. The $\mathrm{WSe}_{2}(\mathrm{~A})$ and $\mathrm{GaN} / \mathrm{WSe} \mathrm{S}_{2}(\mathrm{~B})$ exhibits the direct optical bandgap which is matching with the value of single layer ${ }^{23}$. As represented in Figure $4(\mathrm{~b})$ the observed states $\mathrm{X}_{\mathrm{A}}, \mathrm{X}_{\mathrm{B}}, \mathrm{X}_{\mathrm{A}^{\prime}}$ and $\mathrm{X}_{\mathrm{B}^{\prime}}$ for sample $\mathrm{A}$ at $\approx 1.63$, $\approx 2.08$, $\approx 2.44$ and $\approx 2.91 \mathrm{eV}$ in the absorbance spectrum are the exciton resonances corresponding to the transitions from broken inversion symmetry induced two spin-split valence sub-bands to the conduction band and vice versa ${ }^{36-38}$. Thus, the spin splitting of $\mathrm{WSe}_{2}$ is observed to be $\approx 0.45 \mathrm{eV}$ which is smaller than that of $\mathrm{MoSe}_{2}$ exhibiting the largest value of $\approx 0.55 \mathrm{eV}$ among $\mathrm{MX}_{2}$ family ${ }^{39,40}$. Hence, this observation confirms that the CVD grown $\mathrm{WSe}_{2}$ sample is in the form of SL, as corroborated by earlier AFM, STEM and micro-Raman measurements. However, these are not an exact band to band transitions due to the involvement of excitons. The electronic band gap is measure of actual gap of a material which is the summation of optical bandgap and exciton (electron-hole) binding energy $\left(\mathrm{E}_{\mathrm{g}}=\mathrm{E}_{\mathrm{opt}}+\mathrm{E}_{\mathrm{b}}\right) .{ }^{40}$ Hence, these observed optical bandgap values differ from reported electronic bandgap values of $E_{g}^{S L-W S e_{2}}=2.08 \mathrm{eV}$ and $E_{g}^{G a N}=3.45 \mathrm{eV}$ by their respective exciton binding energy $\left(\mathrm{E}_{\mathrm{b}}\right)$ values of $\approx 0.450$ and $\approx 0.023 \mathrm{eV}$ as reported in the literature..$^{23,41,42}$

High-resolution XPS measurements were extensively used to measure the valence band offset (VBO) of a heterointerface. In order to evaluate VBO at GaN/SL-WSe 2 interface, the energy difference between the $\mathrm{Ga}$ and $\mathrm{W}$ core levels from the GaN/SL- WSe 2 heterojunction sample and the energy of core levels relative to the respective valence band maximum (VBM) of the GaN epilayer and SL- WSe 2 samples need to be acquired. As the escape depth of photo emitted electrons in HRXPS is significantly low, over grown GaN layer of heterojunction sample has to be thin enough so that the electrons removed from both thin overgrown $\mathrm{GaN}$ and underlying SL- WSe 2 layers can be easily examined. ${ }^{43}$ However, the surface area of SL-WSe 2 is large enough to carry out the XPS measurements, due to the non-continuity of the $\mathrm{WSe}_{2}$ film on sapphire substrate, the region of interest on $\mathrm{WSe}_{2} /$ sapphire and $\mathrm{GaN} / \mathrm{WSe}_{2}$ samples was selectively chosen within the 
spatial resolution of HRXPS measurements. ${ }^{23,44}$ This is executed by comparing the intensity of Ga 2p, W $4 \mathrm{f}$ and Al 2p core-levels which allowed us to collect the photoemission signal from solely $\mathrm{SL}_{-\mathrm{WSe}} /$ sapphire and GaN/SL- WSe 2 heterostructures for samples A and B, respectively. Since LMM auger line of $\mathrm{Ga}$ at $\approx 397 \mathrm{eV}$ N overlaps with $\mathrm{N} 1$ s core level for $\mathrm{Al} \mathrm{K}_{\alpha}$ anode as X-ray source in HRXPS measurements, the $\mathrm{N}$ 1s core levels are not considered for the analysis of VBO determination. Furthermore, $\mathrm{Ga} 3 \mathrm{~d}$ is also excluded from the analysis as it exists close to the valence band states. ${ }^{45}$ Thus, in order to determine the VBO, the core levels $\mathrm{W} 4 \mathrm{f}$, Se $3 \mathrm{~d}$ and Ga $2 \mathrm{p}$ were used for the analysis. The valence band offset (VBO) for GaN/SL-WSe 2 heterojunction, can be calculated by the method provided by Kraut et al. ${ }^{46}$, expressed as

$$
\Delta E_{v}=\left(E_{W 4 f_{7 / 2}}^{W S e_{2}}-E_{V B M}^{W S e_{2}}\right)+\left(E_{G a 2 p_{3 / 2}}^{G a N / W S e_{2}}-E_{W 4 f_{7 / 2}}^{G a N / W S e_{2}}\right)-\left(E_{G a 2 p_{3 / 2}}^{G a N}-E_{V B M}^{G a N}\right)
$$

From Figure 5a (i and ii), the first term of equation (1) deduced by taking into account the position of $\mathrm{W} 4 \mathrm{f}_{7 / 2}$ core-level referenced with respect to the VBM, which is measured to be $31.56 \pm 0.10 \mathrm{eV}$. The values of the valence band maxima $(\mathrm{VBM})$ are determined by extrapolating the linear fit to the leading edge of the valence band to the base level. ${ }^{47,48}$ Prior to this, $\mathrm{W} 4 \mathrm{f}$ corelevel is deconvoluted so as to access the accurate value that contributed solely from W-Se bonding in $\mathrm{SL}_{-}-\mathrm{WSe}_{2}$. In this peak deconvolution process, we use Voigt (mixed Lorentzian-Gaussian) line shapes for fitting the $\mathrm{W}-\mathrm{Se}$ (trigonal prismatic $-2 \mathrm{H}$ ) and $\mathrm{W}-\mathrm{O}$ chemical states, respectively, centered at $32.52 \pm 0.10$ and $36.15 \pm 0.10 \mathrm{eV}$. The later state with low intensity results from $\mathrm{WO}_{3}$ precursor or excess $\mathrm{W}$ metal bonding with the oxygen of $\mathrm{c}-\mathrm{Al}_{2} \mathrm{O}_{3}$ at the interface of $\mathrm{WSe} / \mathrm{c}$ sapphire during high temperature CVD growth.

In order to evaluate the subsequent term, GaN/SL-WSe 2 sample is used for XPS measurements where we consider the difference between $\mathrm{Ga} 2 \mathrm{p}_{3 / 2}$ and $\mathrm{W} 4 \mathrm{f}_{7 / 2}$ core-levels. Figure $5 \mathrm{~b}$ (i and ii) depicts the Ga 2p and W 4f core-levels which are acquired on GaN/SL-WSe 2 heterointerface. Figure 5(b-i) represents the Ga 2p core-level which is deconvoluted with Ga-N bonding and any other states are not observed. The Figure 5(b-ii) shows the fitting of $\mathrm{W} 4 \mathrm{f}$ core-level equipped with three $\mathrm{W} 4 \mathrm{f}_{7 / 2}$ chemical states at $30.81 \pm 0.10,31.94 \pm 0.10$ and $36.17 \pm 0.10 \mathrm{eV}$, ascribed to the $\mathrm{W}-\mathrm{Se}$ bonding in $1 \mathrm{~T}-\mathrm{WSe}_{2}$ (octahedral), W-Se bonding in $2 \mathrm{H}-\mathrm{WSe} \mathrm{S}_{2}$ (trigonal prismatic), and $\mathrm{W}-\mathrm{O}$ bonding in $\mathrm{WO}_{3}$, respectively. ${ }^{49}$ The $\mathrm{W}_{4 / 2}$ core-level has similar deconvolutions at higher binding energy values differed by $\approx 2.16 \mathrm{eV}$ from $\mathrm{W} 4 \mathrm{f}_{7 / 2}$ bonding states. The octahedral phase could be due to unintentional N-plasma intercalation of $\mathrm{WSe}_{2}$ single layer during GaN growth which is as similar as lithium intercalation reported for the case of $\mathrm{MoS}_{2}{ }^{37}$. The presence of 
octahedral phase results in the low intensity PL signal for sample B as presented in Figure 4(b). The W-Se bonding peak infers that the sustainability of $\mathrm{SL}_{-} \mathrm{WSe}_{2}$ at $\mathrm{GaN}$ growth temperature under UHV oxygen free environment. The absence of any other chemical state associated with $\mathrm{W}$ or Se in Ga 2p core-level spectrum is a clear evidence of Vander Waal epitaxy. Thus, the energy difference between $\mathrm{W} 4 \mathrm{f}_{7 / 2}$ and $\mathrm{Ga} 2 \mathrm{p}_{3 / 2}$ core-levels is observed to be $1085.92 \pm 0.10 \mathrm{eV}$ as described in Figure 5(b).

The last term indicates the core-level energy $1115.23 \pm 0.10 \mathrm{eV}$ of Ga $2 \mathrm{p}_{3 / 2}$ measured relative to the respective VBM of $2.27 \pm 0.15 \mathrm{eV}$ as shown in Figure 5(c). This VBM measured with respect to the Fermi level for nearly intrinsic GaN which was utilized in the present study is lower than that of the n-type $\mathrm{GaN}$ in the literature $(2.7 \mathrm{eV}) .{ }^{47}$ In Figure $5 \mathrm{c}(\mathrm{i}), \mathrm{Ga} 2 \mathrm{p}_{3 / 2}$ and $2 \mathrm{p}_{1 / 2}$ core-levels are deconvoluted with Ga-N bonding state at $1117.50 \pm 0.10$ and $1144.40 \pm 0.10 \mathrm{eV}$, respectively. The Fermi level position with respect to the VBM as shown in Figure 5(a-ii and c-ii) infers that $\mathrm{GaN}$ epilayer and SL-WSe 2 are nearly intrinsic materials. Thus, the VBO from equation (1) is determined to be $\approx 2.25 \pm 0.15 \mathrm{eV}$. The VBO is also confirmed by using VB spectrum, Se $3 \mathrm{~d}$ and Ga $2 p$ core levels of samples A and B (see supporting information).

As a result, the substitution of VBO $\left(\triangle \mathrm{E}_{\mathrm{v}}\right)$ obtained from HRXPS analysis (Figure 5) and electronic bandgap $\left(\mathrm{E}_{\mathrm{g}}\right)$ values of $\mathrm{SL}-\mathrm{WSe}_{2}$ and $\mathrm{GaN}$ epilayer in equation (2), allows to measure the conduction band offset $(\mathrm{CBO}) \Delta E_{c}$ for GaN/SL-WSe 2 heterostructure.

$$
\Delta E_{c}=E_{g}^{W S e_{2}}+\Delta E_{v}-E_{g}^{G a N}
$$

Thus, the $\mathrm{CBO}\left(\Delta \mathrm{E}_{\mathrm{c}}\right)$ is determined to be $0.88 \pm 0.10 \mathrm{eV}$. Furthermore, the measured $\mathrm{CBO}$ value is verified by the Anderson's affinity rule which is defined as the difference between electron affinity values of constituent semiconductors of heterojuction. ${ }^{50}$ The electron affinity (the energy separation between vacuum level and CBM) values $\chi^{G a N}, \chi^{S L-W S e_{2}}$ of $\mathrm{GaN}$ and SL-WSe 2 were $\approx 4.1$ and $\approx 3.3 \mathrm{eV}$, respectively. ${ }^{51,52}$ UPS measurements were performed to measure the electron affinity of $\mathrm{GaN}$. Here, electron affinity $(\chi)$ of $\mathrm{SL}^{-W S e_{2}}$ is extracted using the formula $\chi=\phi-\left(E_{g}-E_{F}\right)$ from our earlier reported work function value deduced by ultraviolet photoelectron spectroscopic (UPS) measuremetns ${ }^{52}$, where $\phi$ is the work function $(3.61 \mathrm{eV})$ and $\mathrm{E}_{\mathrm{g}}$ is the electronic bandgap $(2.08 \mathrm{eV})$ and $\mathrm{E}_{\mathrm{F}}$ is the Fermi level position with respect to VBM (1.8 $\mathrm{eV}$ ) of an $\mathrm{SL}_{-\mathrm{WSe}}$. For more details see the supporting information. It should be noted that 
electron affinity is material specific and independent of the Fermi level position. Hence, from Anderson's rule, calculated $\mathrm{CBO}$ for GaN/SL-WSe 2 heterojunction is $0.8 \mathrm{eV}$. The determined CBO value of $0.88 \pm 0.15 \mathrm{eV}$ from HRXPS studies in the present study is consistent with the value $(0.8 \mathrm{eV})$ obtained by affinity rule, which facilitates in understanding the electron transport properties across the heterojunction.

Thereby, the experimentally measured offset parameters from this study are presented as a schematic of band discontinuity diagram in Figure 6 which pertains to type-II heterostructure.

\section{CONCLUSIONS}

In conclusion, GaN epitaxial thin layers were deposited on SL-WSe 2 /c-sapphire substrates by PAMBE to study the band discontinuity at GaN/SL-WSe $e_{2}$ heterointerface. We confirm that the CVD deposited $\mathrm{WSe}_{2}$ is a single-layer by complementary characterization tools such as AFM, HAADF-STEM, micro-Raman modes, absorbance and $\mu \mathrm{PL}$ studies. The determination of band offset parameters at GaN/SL-WSe 2 heterostructure was carried out by utilizing the HRXPS, electron affinities and electronic band gap values. We determine the valence band and conduction band offset values, respectively, of $2.25 \pm 0.15 \mathrm{eV}$ and $0.8 \pm 0.15 \mathrm{eV}$ with type II band alignment at GaN/SL-WSe 2 heterostructure. This demonstration of unprecedented band offset parameters renders a route towards the integration of $3 \mathrm{D}$ group III nitride materials with $2 \mathrm{D}$ transition metal dichalcogenide layers.

\section{ASSOCIATED CONTENT}

Supporting Information: Confirmation of valence band offset by using Ga 2p and Se 3d HRXPS core levels. Determination of electron affinity for $\mathrm{GaN}$ and $\mathrm{SL}-\mathrm{WSe} 2$ by ultraviolet photoelectron spectroscopy (UPS).

\section{AUTHOR INFORMATION}

Corresponding author:

*Email: boon.ooi@kaust.edu.sa

Notes The authors declare no competing financial interest. 


\section{ACKNOWLEDGEMENTS}

We acknowledge the financial support from King Abdulaziz City for Science and Technology (KACST), Grant No. KACST TIC R2-FP-008 and baseline funding BAS/1/1614-0101 of the King Abdullah University of Science and Technology (KAUST).

\section{REFERENCES}

(1) Nakamura, S.; Senoh, M.; Nagahama, S.; Iwasa, N.; Yamada, T.; Matsushita, T.; Kiyoku, H.; Sugimoto, Y.; Kozaki, T.; Umemoto, H.; Sano, M.; Chocho, K. Violet InGaN/GaN/AlGaN-Based Laser Diodes with an Output Power of 420 mW. Jpn. J. Appl. Phys. 1998, 37 (Part 2, No. 6A), L627-L629.

(2) Shen, L.; Heikman, S.; Moran, B.; Coffie, R.; Zhang, N.-Q.; Buttari, D.; Smorchkova, I. P.; Keller, S.; DenBaars, S. P.; Mishra, U. K. AlGaN/AlN/GaN High-Power Microwave HEMT. IEEE Electron Device Lett. 2001, 22 (10), 457-459.

(3) Zhao, C.; Ng, T. K.; Elafandy, R. T.; Prabaswara, A.; Consiglio, G. B.; Ajia, I. A.; Roqan, I. S.; Janjua, B.; Shen, C.; Eid, J.; Alyamani, A. Y.; El-Desouki, M. M.; Ooi, B. S. DroopFree, Reliable, and High-Power InGaN/GaN Nanowire Light-Emitting Diodes for Monolithic Metal-Optoelectronics. Nano Lett. 2016, 16 (7), 4616-4623.

(4) Tangi, M.; De, A.; Ghatak, J.; Shivaprasad, S. M. Electron Mobility of Self-Assembled and Dislocation Free InN Nanorods Grown on GaN Nano Wall Network Template. J. Appl. Phys. 2016, 119 (May), 205701.

(5) Radisavljevic, B.; Radenovic, A.; Brivio, J.; Giacometti, V.; Kis, A. Single-Layer MoS2 Transistors. Nat. Nanotechnol. 2011, 6 (3), 147-150.

(6) Baugher, B. W. H.; Churchill, H. O. H.; Yang, Y.; Jarillo-Herrero, P. Optoelectronic Devices Based on Electrically Tunable P-N Diodes in a Monolayer Dichalcogenide. Nat. Nanotechnol. 2014, 9 (4), 262.

(7) Pu, J.; Kanahashi, K.; Cuong, N. T.; Chen, C.-H.; Li, L.-J.; Okada, S.; Ohta, H.; Takenobu, T. Enhanced Thermoelectric Power in Two-Dimensional Transition Metal Dichalcogenide Monolayers. Phys. Rev. B 2016, 94 (1), 14312.

(8) Li, M.-Y.; Shi, Y.; Cheng, C.-C.; Lu, L.-S.; Lin, Y.-C.; Tang, H.-L.; Tsai, M.-L.; Chu, C.W.; Wei, K.-H.; He, J.-H.; Chang, W.-H.; Suenaga, K.; Li, L.-J. Epitaxial Growth of a Monolayer $\mathrm{WSe}_{2}-\mathrm{MoS}_{2}$ Lateral P-N Junction with an Atomically Sharp Interface. Science 
(80-. ). 2015, 349 (6247), 524.

(9) Eichfeld, S. M.; Hossain, L.; Lin, Y.; Piasecki, A. F.; Kupp, B.; Birdwell, A. G.; Burke, R. A.; Lu, N.; Peng, X.; Li, J.; Azcatl, A.; McDonnell, S.; Wallace, R. M.; Kim, M. J.; Mayer, T. S.; Redwing, J. M.; Robinson, J. A. Highly Scalable, Atomically Thin $\mathrm{WSe}_{2}$ Grown via Metal-Organic Chemical Vapor Deposition. ACS Nano 2015, 9 (2), 2080-2087.

(10) Huo, N.; Tongay, S.; Guo, W.; Li, R.; Fan, C.; Lu, F.; Yang, J.; Li, B.; Li, Y.; Wei, Z. Novel Optical and Electrical Transport Properties in Atomically Thin $\mathrm{WSe}_{2} / \mathrm{MoS}_{2} \mathrm{P}-\mathrm{N}$ Heterostructures. Adv. Electron. Mater. 2015, 1 (5), 1400066.

(11) Gupta, P.; Rahman, A. A.; Subramanian, S.; Gupta, S.; Thamizhavel, A.; Orlova, T.; Rouvimov, S.; Vishwanath, S.; Protasenko, V.; Laskar, M. R.; Xing, H. G.; Jena, D.; Bhattacharya, A. Layered Transition Metal Dichalcogenides: Promising near-LatticeMatched Substrates for GaN Growth. Sci. Rep. 2016, 6 (March), 23708.

(12) Reshchikov, M. A.; Morkoç, H. Luminescence Properties of Defects in GaN. J. Appl. Phys. 2005, 97 (2005), 61301.

(13) Liu, L.; Edgar, J. H. Substrates for Gallium Nitride Epitaxy. Mater. Sci. Eng. R Reports 2002, 37 (3), 61-127.

(14) II, E. W. L.; Lee, C. H.; Paul, P. K.; Ma, L.; McCulloch, W. D.; Krishnamoorthy, S.; Wu, Y.; Arehart, A. R.; Rajan, S. Layer-Transferred $\mathrm{MoS}_{2} / \mathrm{GaN}$ PN Diodes. Appl. Phys. Lett. 2015, 107 (10), 103505.

(15) Krishnamoorthy, S.; Lee, E. W.; Lee, C. H.; Zhang, Y.; McCulloch, W. D.; Johnson, J. M.; Hwang, J.; Wu, Y.; Rajan, S. High Current Density 2D/3D Esaki Tunnel Diodes. Appl. Phys. Lett. 2016, 183505 (June), 183505.

(16) Yamada, A.; Ho, K. P.; Maruyama, T.; Akimoto, K. Molecular Beam Epitaxy of GaN on a Substrate of $\mathrm{MoS}_{2}$ Layered Compound. Appl. Phys. A Mater. Sci. Process. 1999, 69 (1), 89-92.

(17) Mishra, P.; Tangi, M.; Ng, T. K.; Hedhili, M. N.; Anjum, D. H.; Alias, M. S.; Tseng, C.-C.; Li, L.-J.; Ooi, B. S. Impact of N-Plasma and Ga-Irradiation on $\mathrm{MoS}_{2}$ Layer in Molecular Beam Epitaxy. Appl. Phys. Lett. 2017, 110 (1), 12101.

(18) Ruzmetov, D.; Zhang, K.; Stan, G.; Kalanyan, B.; Bhimanapati, G. R.; Eichfeld, S. M.; Burke, R. A.; Shah, P. B.; O’Regan, T. P.; Crowne, F. J.; Birdwell, A. G.; Robinson, J. A.; Davydov, A. V; Ivanov, T. G. Vertical 2D/3D Semiconductor Heterostructures Based on 
Epitaxial Molybdenum Disulfide and Gallium Nitride. ACS Nano 2016, 10, 3580-3588.

(19) Huang, J. K.; Pu, J.; Hsu, C. L.; Chiu, M. H.; Juang, Z. Y.; Chang, Y. H.; Chang, W. H.; Iwasa, Y.; Takenobu, T.; Li, L. J. Large-Area Synthesis of Highly Crystalline WSe 2 Monolayers and Device Applications. ACS Nano 2014, 8 (1), 923-930.

(20) King, P. D. C.; Veal, T. D.; Kendrick, C.; Bailey, L.; Durbin, S.; McConville, C. F. InN/GaN Valence Band Offset: High-Resolution X-Ray Photoemission Spectroscopy Measurements. Phys. Rev. B 2008, 78 (3), 33308.

(21) Martin, G.; Strite, S.; Botchkarev, A.; Agarwal, A.; Rockett, A.; Morkoç, H.; Lambrecht, W. R. L.; Segall, B. Valence-Band Discontinuity between GaN and AIN Measured by XRay Photoemission Spectroscopy. Appl. Phys. Lett. 1994, 65 (5), 610-612.

(22) Bhat, T. N.; Kumar, M.; Rajpalke, M. K.; Roul, B.; Krupanidhi, S. B.; Sinha, N. Band Alignment Studies in InN/p-Si(100) Heterojunctions by X-Ray Photoelectron Spectroscopy. J. Appl. Phys. 2011, 109 (12), 123707.

(23) Chiu, M.-H.; Zhang, C.; Shiu, H.-W.; Chuu, C.-P.; Chen, C.-H.; Chang, C.-Y. S.; Chen, C.H.; Chou, M.-Y.; Shih, C.-K.; Li, L.-J. Determination of Band Alignment in the SingleLayer $\mathrm{MoS}_{2} / \mathrm{WSe}_{2}$ Heterojunction. Nat. Commun. 2015, 6 (May), 7666.

(24) Siol, S.; Hellmann, J. C.; Tilley, S. D.; Graetzel, M.; Morasch, J.; Deuermeier, J.; Jaegermann, W.; Klein, A. Band Alignment Engineering at $\mathrm{Cu}_{2} \mathrm{O} / \mathrm{ZnO}$ Heterointerfaces. ACS Appl. Mater. Interfaces 2016, 8 (33), 21824-21831.

(25) Qiao, L.; Li, W.; Xiao, H.; Meyer, H. M.; Liang, X.; Nguyen, N. V.; Weber, W. J.; Biegalski, M. D. Electronic Structure and Band Alignment at an Epitaxial Spinel/perovskite Heterojunction. ACS Appl. Mater. Interfaces 2014, 6 (16), 14338-14344.

(26) Tangi, M.; Mishra, P.; Ng, T. K.; Hedhili, M. N.; Janjua, B.; Alias, M. S.; Anjum, D. H.; Tseng, C. C.; Shi, Y.; Joyce, H. J.; Li, L. J.; Ooi, B. S. Determination of Band Offsets at GaN/single-Layer MoS 2 Heterojunction. Appl. Phys. Lett. 2016, 109 (3), 32104.

(27) Bersch, E.; Rangan, S.; Bartynski, R. A.; Garfunkel, E.; Vescovo, E. Band Offsets of Ultrathin High- K Oxide Films with Si. Phys. Rev. B 2008, 78, 85114.

(28) King, S. W.; Paquette, M. M.; Otto, J. W.; Caruso, A. N.; Brockman, J.; Bielefeld, J.; Kuhn, M.; French, B.; King, S. W.; Paquette, M. M.; Otto, J. W.; Caruso, A. N.; Brockman, J.; Bielefeld, J.; French, M.; Kuhn, M.; French, B. Valence and Conduction Band Offsets at Amorphous Hexagonal Boron Nitride Interfaces with Silicon Network Dielectrics. Appl. 
Phys. Lett. 2014, 104, 102901.

(29) Distefano, J.; Lin, Y.; Robinson, J.; Glavin, N. R.; Voevodin, A. A.; Brockman, J.; Kuhn, M.; French, B.; King, S. W. Band Alignment at Molybdenum Disulphide / Boron Nitride / Aluminum Oxide Interfaces. J. Electron. Mater. 2016, 45 (2), 983-988.

(30) Tangi, M.; Mishra, P.; Janjua, B.; Ng, T. K.; Anjum, D. H.; Prabaswara, A.; Yang, Y.; Albadri, A. M.; Alyamani, A. Y.; El-Desouki, M. M.; Ooi, B. S. Bandgap Measurements and the Peculiar Splitting of E2H Phonon Modes of $\operatorname{In}_{\mathrm{x}} \mathrm{Al}_{1-\mathrm{x}} \mathrm{N}$ Nanowires Grown by Plasma Assisted Molecular Beam Epitaxy. J. Appl. Phys. 2016, 120 (4), 45701.

(31) Chakraborty, B.; Bera, A.; Muthu, D. V. S.; Bhowmick, S.; Waghmare, U. V.; Sood, A. K. Symmetry-Dependent Phonon Renormalization in Monolayer $\mathrm{MoS}_{2}$ Transistor. Phys. Rev. $B$ 2012, 85 (16), 161403(R).

(32) Huang, J.; Yang, L.; Liu, D.; Chen, J.; Fu, Q.; Xiong, Y.; Lin, F.; Xiang, B. Large-Area Synthesis of Monolayer $\mathrm{WSe}_{2}$ on a $\mathrm{SiO}_{2} / \mathrm{Si}$ Substrate and Its Device Applications. Nanoscale 2015, 7 (9), 4193-4198.

(33) Zeng, H.; Liu, G.-B.; Dai, J.; Yan, Y.; Zhu, B.; He, R.; Xie, L.; Xu, S.; Chen, X.; Yao, W.; Cui, X. Optical Signature of Symmetry Variations and Spin-Valley Coupling in Atomically Thin Tungsten Dichalcogenides. Sci. Rep. 2013, 3 (4), 1608.

(34) Sugawara, K.; Sato, T.; Tanaka, Y.; Souma, S.; Takahashi, T. Spin- and Valley-Coupled Electronic States in Monolayer WSe 2 on Bilayer Graphene. Appl. Phys. Lett. 2015, 107 (7), 71601.

(35) del Corro, E.; Botello-Méndez, A.; Gillet, Y.; Elias, A. L.; Terrones, H.; Feng, S.; Fantini, C.; Rhodes, D.; Pradhan, N.; Balicas, L.; Gonze, X.; Charlier, J.-C.; Terrones, M.; Pimenta, M. A. Atypical Exciton-Phonon Interactions in $\mathrm{WS}_{2}$ and $\mathrm{WSe}_{2}$ Monolayers Revealed by Resonance Raman Spectroscopy. Nano Lett. 2016, 16, 2363.

(36) Mak, K. F.; Shan, J. Photonics and Optoelectronics of 2D Semiconductor Transition Metal Dichalcogenides. Nat. Photonics 2016, 10 (4), 216-226.

(37) Eda, G.; Yamaguchi, H.; Voiry, D.; Fujita, T.; Chen, M.; Chhowalla, M. Photoluminescence from Chemically Exfoliated $\mathrm{MoS}_{2}$. Nano Lett. 2011, 11 (12), 5111-5116.

(38) Jin, W.; Yeh, P. C.; Zaki, N.; Zhang, D.; Sadowski, J. T.; Al-Mahboob, A.; Van Der Zande, A. M.; Chenet, D. A.; Dadap, J. I.; Herman, I. P.; Sutter, P.; Hone, J.; Osgood, R. M. Direct Measurement of the Thickness-Dependent Electronic Band Structure of $\mathrm{MoS}_{2}$ Using Angle- 
Resolved Photoemission Spectroscopy. Phys. Rev. Lett. 2013, 111 (10), 106801.

Zhu, Z. Y.; Cheng, Y. C.; Schwingenschlögl, U. Giant Spin-Orbit-Induced Spin Splitting in Two-Dimensional Transition-Metal Dichalcogenide Semiconductors. Phys. Rev. B 2011, $84(15), 153402$.

(40) Ugeda, M. M.; Bradley, A. J.; Shi, S.-F.; da Jornada, F. H.; Zhang, Y.; Qiu, D. Y.; Ruan, W.; Mo, S.-K.; Hussain, Z.; Shen, Z.-X.; Wang, F.; Louie, S. G.; Crommie, M. F. Giant Bandgap Renormalization and Excitonic Effects in a Monolayer Transition Metal Dichalcogenide Semiconductor. Nat. Mater. 2014, 13 (12), 1091-1095.

(41) Zhou, S. Q.; Wu, M. F.; Hou, L. N.; Yao, S. D.; Ma, H. J.; Nie, R.; Tong, Y. Z.; Yang, Z. J.; Yu, T. J.; Zhang, G. Y. An Approach to Determine the Chemical Composition in InGaN/GaN Multiple Quantum Wells. J. Cryst. Growth 2004, 263 (1-4), 35-39.

(42) Muth, J. F.; Lee, J. H.; Shmagin, I. K.; Kolbas, R. M.; Casey, H. C.; Keller, B. P.; Mishra, U. K.; DenBaars, S. P. Absorption Coefficient, Energy Gap, Exciton Binding Energy, and Recombination Lifetime of GaN Obtained from Transmission Measurements. Appl. Phys. Lett. 1997, 71 (18), 2572.

(43) Fadley, C. S. Atomic-Level Characterization of Materials with Core- and Valence-Level Photoemission: Basic Phenomena and Future Directions. Surf. Interface Anal. 2008, 40 (13), 1579-1605.

(44) Choi, J.; Zhang, H.; Choi, J. H. Modulating Optoelectronic Properties of Two-Dimensional Transition Metal Dichalcogenide Semiconductors by Photoinduced Charge Transfer. ACS Nano 2016, 10, 1671-1680.

(45) Zanatta, A. R.; Hammer, P.; Alvarez, F. Photoelectron Spectroscopic Study of Amorphous GaAsN Films. Appl. Phys. Lett. 2000, 76 (16), 2211.

(46) Kraut, E. A.; Grant, R. W.; Waldrop, J. R.; Kowalczyk, S. P. Precise Determination of the Valence-Band Edge in X-Ray Photoemission Spectra: Application to Measurement of Semiconductor Interface Potentials. Phys. Rev. Lett. 1980, 44 (24), 1620-1623.

(47) King, S. W.; Ronning, C.; Davis, R. F.; Benjamin, M. C.; Nemanich, R. J. Dependence of (0001) GaN/AlN Valence Band Discontinuity on Growth Temperature and Surface Reconstruction. J. Appl. Phys. 1998, 84 (4), 2086-2090.

(48) Tangi, M.; Kuyyalil, J.; Shivaprasad, S. M. Optical Bandgap and near Surface Band Bending in Degenerate InN Films Grown by Molecular Beam Epitaxy. J. Appl. Phys. 2013, 
$114(15), 153501$.

(49) Ambrosi, A.; Sofer, Z.; Pumera, M. 2H $\rightarrow$ 1T Phase Transition and Hydrogen Evolution Activity of $\mathrm{MoS}_{2}, \mathrm{MoSe}_{2}, \mathrm{WS}_{2}$ and $\mathrm{WSe}_{2}$ Strongly Depends on the $\mathrm{MX}_{2}$ Composition. Chem. Commun. 2015, 51 (40), 8450.

(50) Anderson, R. L. Germanium-Gallium Arsenide Heterojunctions [Letter to the Editor]. IBM J. Res. Dev. 1960, 4 (3), 283-287.

(51) Bougrov, V.; Levinshtein, M. E.; Rumyantsev, S. L.; Zubrilov, A. Properties of Advanced Semiconductor Materials: GaN, AlN, InN, BN, SiC, SiGe; Levinshtein, M. E., Rumyantsev, S. L., Shur, M. S., Eds.; John Wiley \& Sons, Inc.: New York, 2001.

(52) Chen, C.-H.; Wu, C.-L.; Pu, J.; Chiu, M.-H.; Kumar, P.; Takenobu, T.; Li, L.-J. Hole Mobility Enhancement and P -Doping in Monolayer WSe $\mathrm{W}_{2}$ by Gold Decoration. 2D Mater. 2014, 1 (3), 34001. 
Figures and captions.
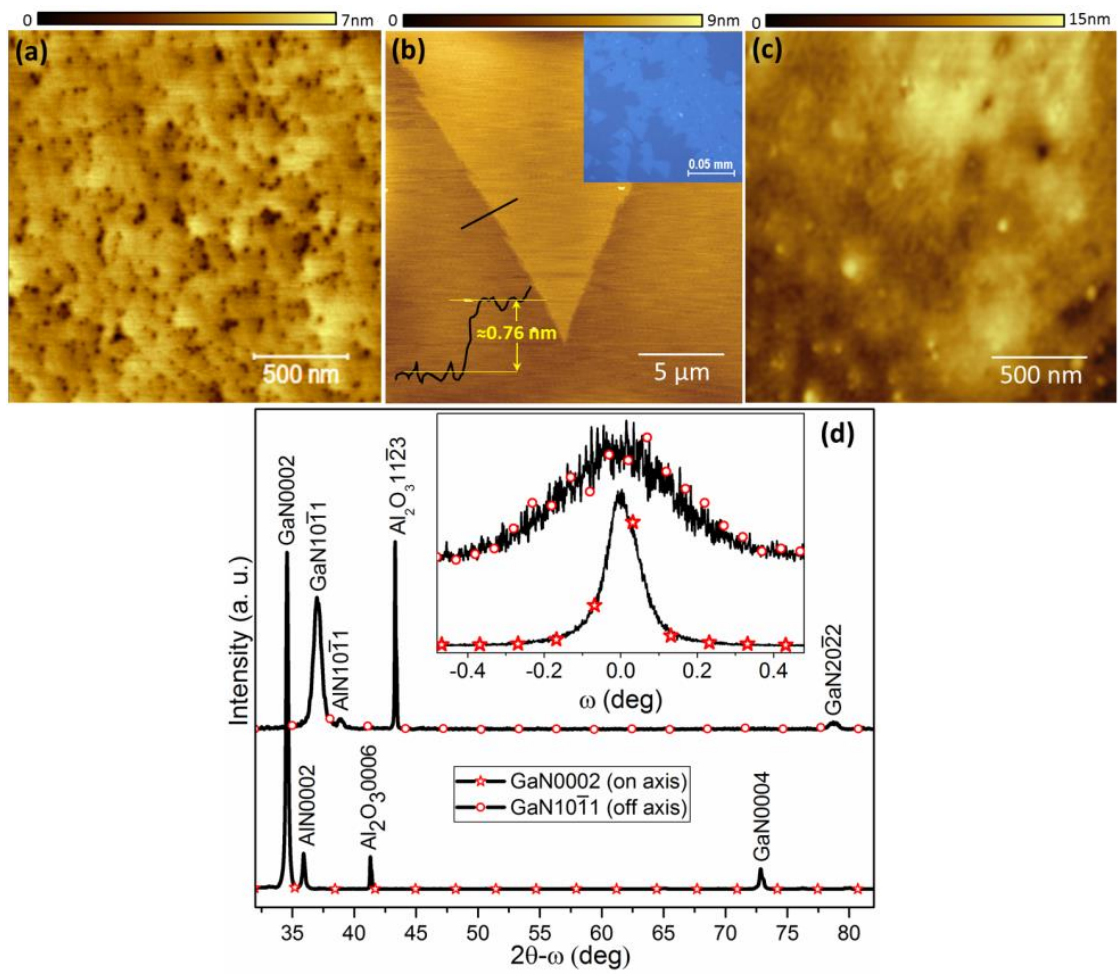

Figure 1 shows the AFM scans for (a) GaN epilayer, (b) WSe 2 and (c) GaN/WSe 2 samples, respectively. The line profile of 1 (b) represents the thickness of $\mathrm{WSe}_{2}$ layer. The inset to Figure

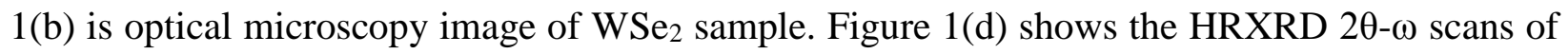
on-axis and off-axis reflections and the inset shows the respective $\omega$-scans acquired on GaN epilayer. 


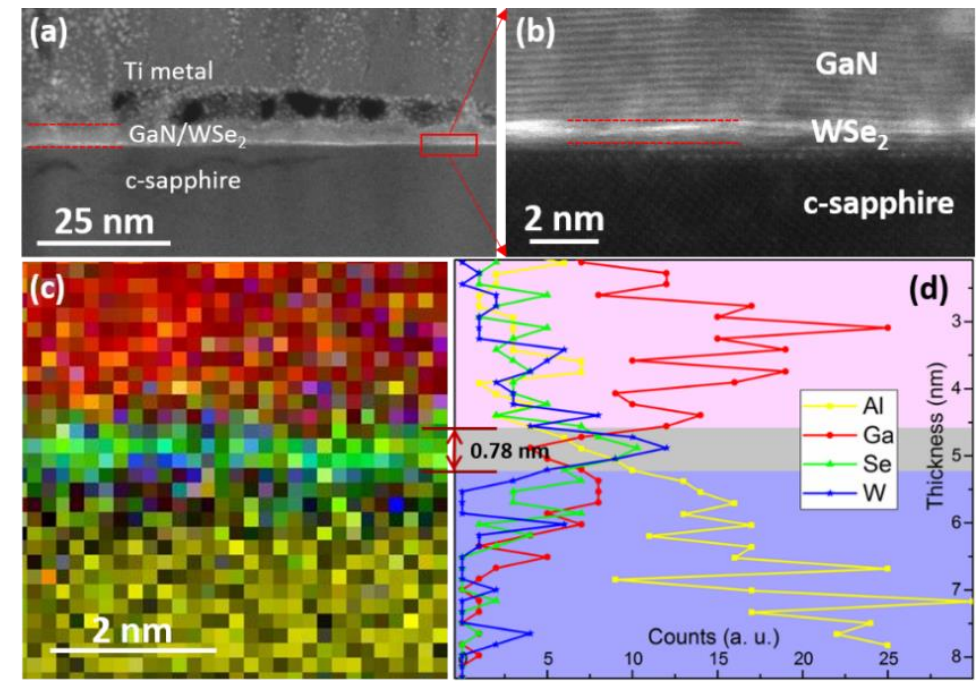

Figure 2 ( $\mathrm{a}$ and $\mathrm{b}$ ) show the low and high magnification cross-sectional images of high-angle annular dark field - scanning transmission electron microscopy (HAADF-STEM) for sample B. (c) shows the STEM-EELS elemental color maps (W-Blue, Se-Green, Ga-Red, and Al-Yellow). Figure 2(d) represents the STEM-EDS elemental line profiles acquired across the GaN/WSe $2 / c-$ sapphire heterojunction. 


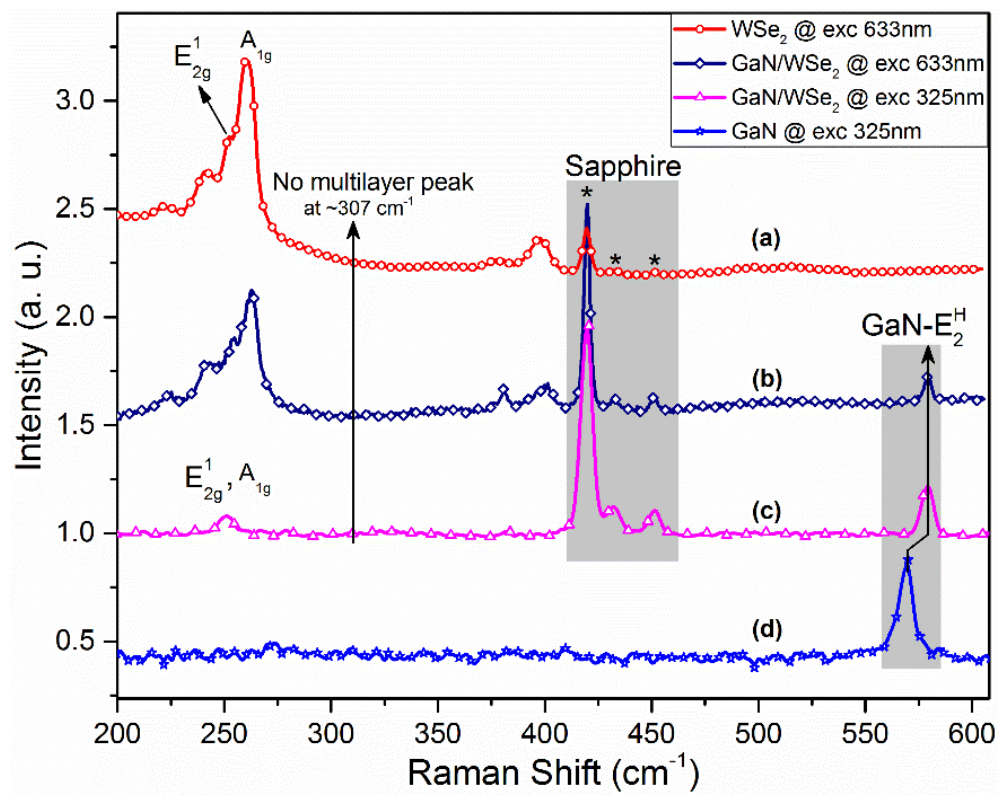

Figure 3(a-d) show the micro-Raman spectra of samples A-C collected on $\mathrm{WSe}_{2}, \mathrm{GaN} / \mathrm{WSe} \mathrm{C}_{2}$ and bulk like GaN samples, respectively. Here, He-Cd $(325 \mathrm{~nm})$ and He-Ne $(633 \mathrm{~nm})$ excitation sources $\left(E_{\text {exc }}>E_{g}\right)$ were used to enhance the Raman signal based on the band gap of each material. 


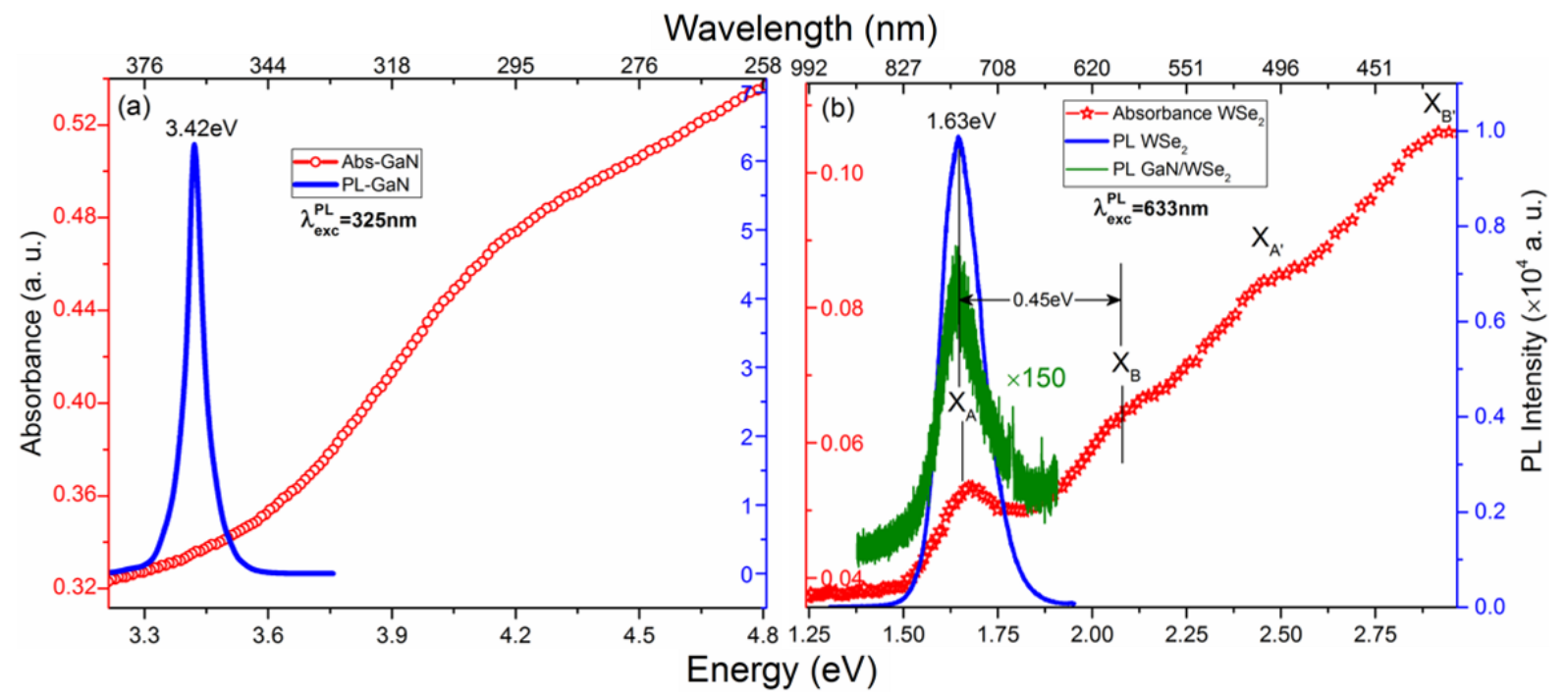

Figure 4(a and b) show the micro-photoluminescence (right y-axis) and absorbance spectra (left $\mathrm{y}$-axis) of $\mathrm{GaN}$ and $\mathrm{WSe}_{2}$ samples. The green colored PL peak in Figure 4(b) is for GaN/WSe 2 sample. 


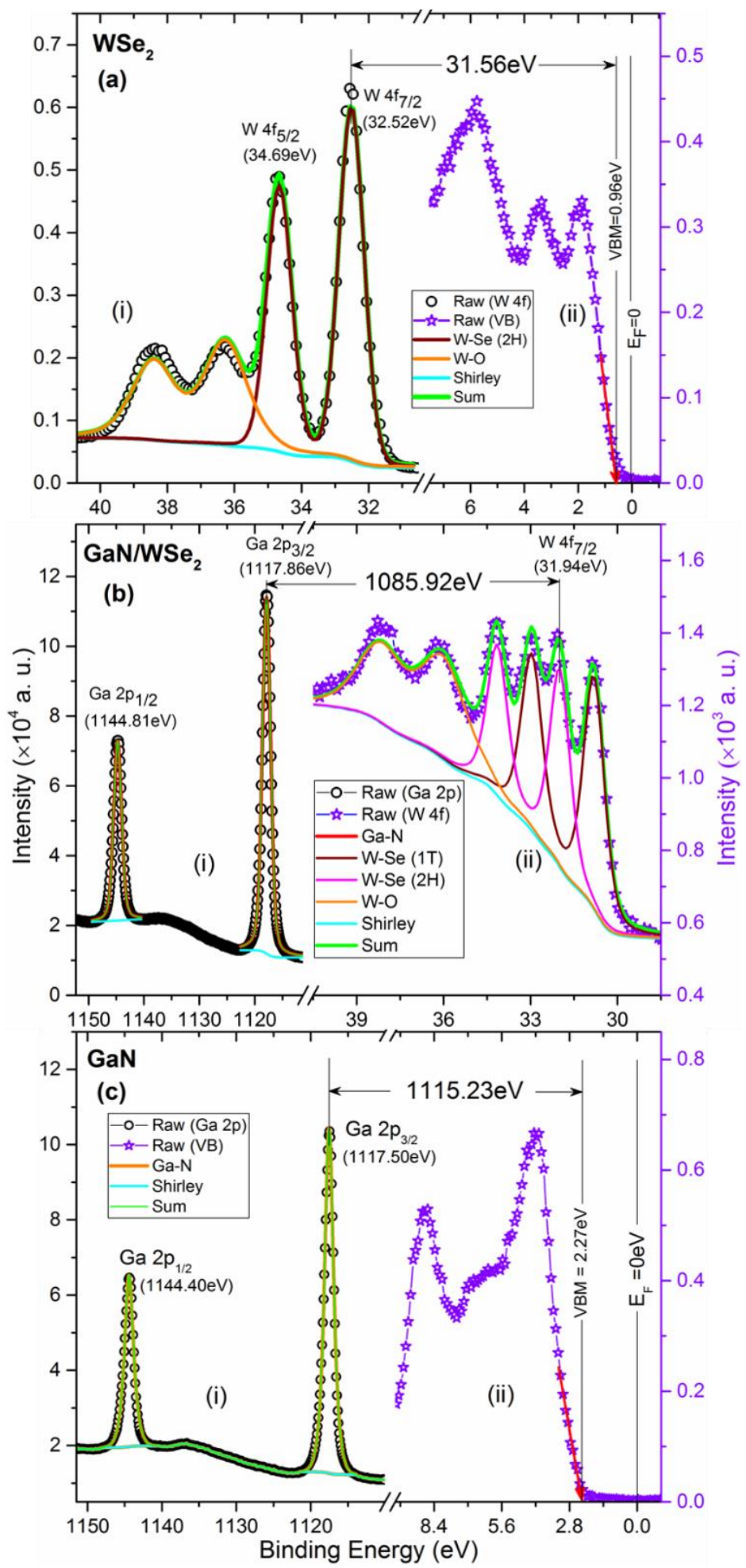

Figure 5a (i and ii) present the $\mathrm{W} 4 \mathrm{f}$ core-level and valence band spectra for SL-WSe 2 . b(i and ii) show the spectra of Ga $2 p$ and W $4 f$ core-levels for GaN/SL-WSe 2 . c(i and ii) show Ga $2 p$ corelevel and valence band spectra acquired on GaN epilayer. The peak positions of core-levels are shown in parentheses. 


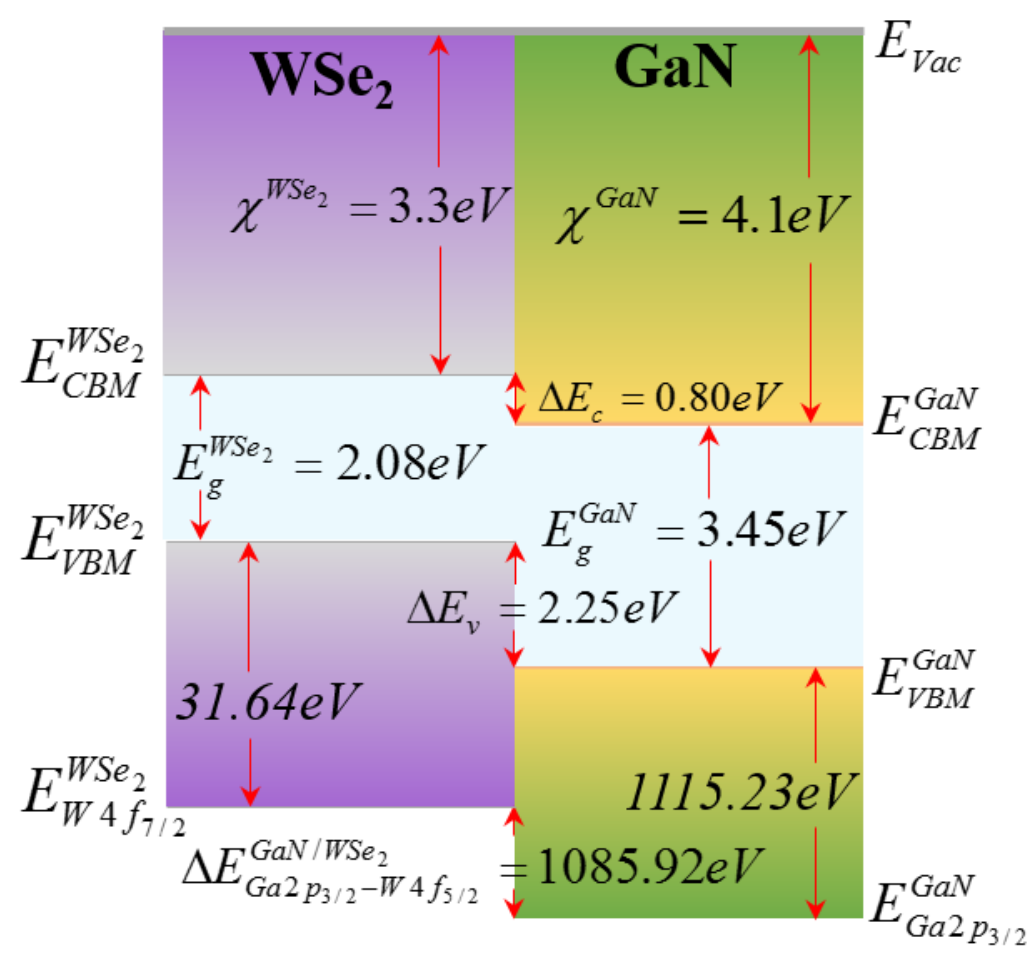

Figure 6 shows the schematic representation of type II band alignment at GaN/SL-WSe 2 heterointerface. 
TOC

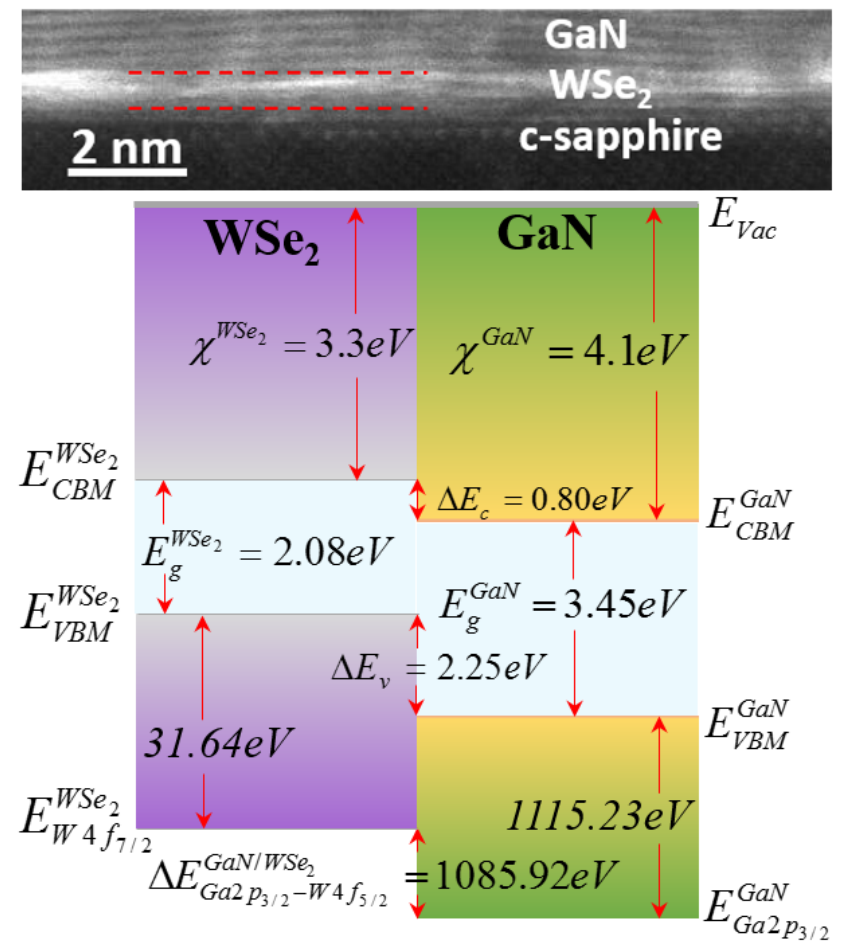

